\title{
Comparison of DC Bead-irinotecan and DC Bead-topotecan drug eluting beads for use in locoregional drug delivery to treat pancreatic cancer
}

\author{
Richard E. J. Forster · Sharon A. Small • Yiqing Tang • \\ Clare L. Heaysman · Andrew W. Lloyd · Wendy Macfarlane • \\ Gary J. Phillips • Milan D. Antonijevic • Andrew L. Lewis
}

Received: 11 January 2010/Accepted: 24 May 2010/Published online: 19 June 2010

(C) The Author(s) 2010. This article is published with open access at Springerlink.com

\begin{abstract}
DC $\mathrm{Bead}^{\circledR}$ is a drug delivery embolisation system that can be loaded with doxorubicin or irinotecan for the treatment of a variety of liver cancers. In this study we demonstrate that the topoisomerase I inhibitor topotecan hydrochloride can be successfully loaded into the DC Bead sulfonate-modified polyvinyl alcohol hydrogel matrix, resulting in a sustained-release drug eluting bead (DEBTOP) useful for therapeutic purposes. The in vitro drug loading capacity, elution characteristics and the effects on mechanical properties of the beads are described with reference to our previous work with irinotecan hydrochloride (DEBIRI). Results showed that drug loading was faster when the solution was agitated compared to static loading and a maximum loading of ca. $40-45 \mathrm{mg}$ topotecan in $1 \mathrm{ml}$ hydrated beads was achievable. Loading the drug into the beads altered the size, compressibility moduli and colour of the bead. Elution was shown to be reliant on the presence of ions to perform the necessary exchange with the electrostatically bound topotecan molecules. Topotecan was shown by MTS assay to have an $\mathrm{IC}_{50}$ for human pancreatic adenocarcinoma cells (PSN-1) of 0.22 and $0.27 \mu \mathrm{M}$ compared to 28.1 and $19.2 \mu \mathrm{M}$ for
\end{abstract}

R. E. J. Forster · S. A. Small · Y. Tang

C. L. Heaysman · A. L. Lewis ( $\square)$

Biocompatibles UK Ltd., Farnham Business Park,

Weydon Lane, Farnham, Surrey GU9 8QL, UK

e-mail: andrew.lewis@biocompatibles.com

R. E. J. Forster · A. W. Lloyd · W. Macfarlane - G. J. Phillips Biomedical Materials Research Group, School of Pharmacy and Biomolecular Sciences, University of Brighton, Mouslecoomb, Brighton, E. Sussex BN2 4GJ, UK

M. D. Antonijevic

The University of Greenwich at Medway, Central Avenue, Chatham Maritime, Kent ME4 4TB, UK irinotecan at 48 and $72 \mathrm{~h}$, respectively. The cytotoxic efficacy of DEBTOP on PSN-1 was compared to DEBIRI. DEPTOP loaded at $6 \& 30 \mathrm{mg} \mathrm{m}^{-1}$, like its free drug form, was shown to be more potent than DEBIRI of comparable doses at $24,48 \& 72 \mathrm{~h}$ using a slightly modified MTS assay. Using a PSN-1 mouse xenograft model, DEBIRI doses of 3.3-6.6 mg were shown to be welltolerated (even with repeat administration) and effective in reducing the tumour size. DEBTOP however, was lethal after 6 days at doses of $0.83-1.2 \mathrm{mg}$ but demonstrated reasonable efficacy and tolerability (again with repeat injection possible) at $0.2-0.4 \mathrm{mg}$ doses. Care must therefore be taken when selecting the dose of topotecan to be loaded into DC Bead given its greater potency and potential toxicity.

\section{Introduction}

Topotecan is a semi-synthetic analogue of camptothecin, a chemotherapeutic agent derived from Camptothecan acuminata, the oriental yew. The cytotoxic mode of action of this family of compounds is understood to be related to their ability to bind and inhibit the enzyme type 1 topoisomerase (Topo-1), which is involved in the replication and repair of DNA during the S-phase of the cell cycle [1]. As DNA is replicated in dividing cells, Topo- 1 acts by binding to super-coiled DNA and causing controlled single-stranded breaks which relieve the torsional stresses that are introduced into DNA ahead of the replication complex or moving replication fork. Topotecan inhibits Topo-1 by stabilizing the covalent complex of enzyme and strandcleaved DNA, inducing uncontrolled single and double strand breaks in the DNA and damage to the replication fork which results in cell death. Topotecan is expected to 
possess a degree of selectivity in its cytotoxic action, as cell division is more frequent and there are higher levels of Topo-1 in malignant cells compared to normal cells [2]. Its chemical structure consists of a fused ring structure containing a lactone ring which is vital to its Topo-1 binding action. The lactone is hydrolysed at neutral $\mathrm{pH}$ and above to form the open-ring carboxylate which is inactive; hence, it is important to the activity of any formulation of this drug that the closed ring lactone form is maintained.

DC Bead has been recently commercialised and used successfully in the clinic for the local treatment of hepatocellular carcinoma [3-5], various neuroendocrine metastases [6] and colorectal cancer metastases to the liver [7, 8] by transarterial chemoembolisation (TACE) of the feeding arteries of the malignancies. More recent preclinical studies have shown that it is feasible to administer DC Bead by other routes, for instance by intraperitoneal (IP) injection for the treatment of peritoneal carcinomatosis [9], or by direct intratumoral (IT) injection as a therapy for glioblastoma multiforme [10]. The DC Bead is based upon a sulfonate-modified polyvinyl alcohol hydrogel, which permits loading and subsequent delivery of variety of positivelycharged chemotherapeutic agents including doxorubicin, mitoxantrone and irinotecan [11-13]. The beads are available in a number of size ranges from $100-300 \mu \mathrm{m}$, 300-500 $\mu \mathrm{m}$ and 500-700 $\mu \mathrm{m}$, with the two smaller size ranges being most popular for embolisation of tumours. It has been demonstrated that affinity between drug and bead is promoted by the ionic interaction of their opposite charges. In the case of irinotecan (another water-soluble camptothecin derivative), the lactone ring formation is maintained intact by this ionic interaction which drives the equilibrium in favour of the positively-charged lactone form over the zwitterionic carboxylate [14].

Topotecan is available as a formulated product known commercially as Hycamptin ${ }^{\circledR}$ containing as the active ingredient topotecan hydrochloride. It is positively charged by virtue of the pendent dimethylamine hydrochloride salt, which differs somewhat to that of the salt formed on the dipiperidino ring of irinotecan. It does, however, possess the same lactone-ring structure as irinotecan and could be expected to benefit from the same mode of preservation of the active form [14] (Fig. 1). Irinotecan is a prodrug that requires the cleavage of the dipiperidino side chain by carboxylesterases present in the body to generate the more potent active metabolite $\mathrm{SN}-38$. Topotecan does not require this enzymatic activation and is therefore used in very much lower doses (typically $1-2 \mathrm{mg} \mathrm{m}^{-2}$ compared to several hundred $\mathrm{mg} \mathrm{m}^{-2}$ for irinotecan [15]). The early uses of topotecan as a chemotherapeutic agent have been in the treatment of ovarian cancer and small cell lung cancer (SCLC) but studies have also been performed in the treatment of brain tumours [16]. Its systemic use has significant

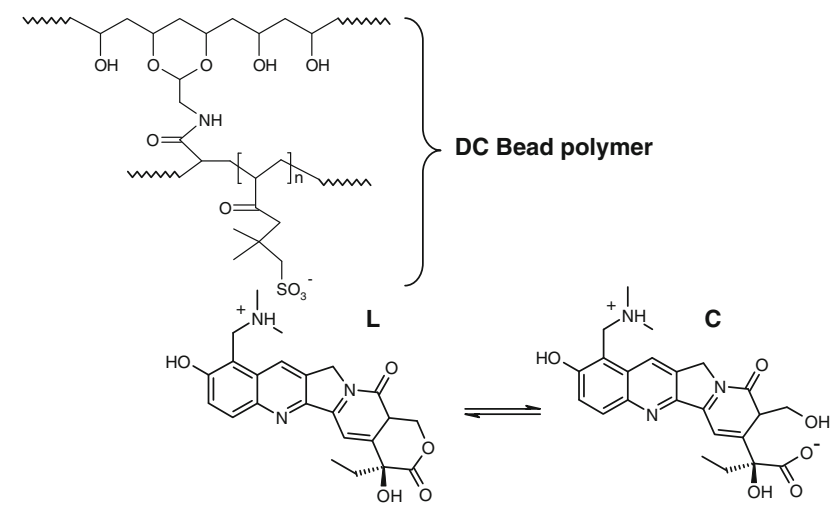

Fig. 1 Chemical structure of DC Bead and its proposed interaction with the active lactone (L) form of topotecan. The active lactone form is believed to bind to the DEBs and elute in this form. Once eluted topotecan can behave like free topotecan and converts to the more stable inactive carboxylate $(\mathrm{C})$ form

side effects including diarrhoea, neutropenia and increased susceptibility to infection. It therefore possesses several attributes that make it an excellent candidate to consider for local delivery using DEB: high potency but with systemic toxicity; active against a range of cancer types; positively charged salt for ionic interaction; available in a formulation that could be used to load the drug immediately prior to a procedure (as is currently performed using DC Bead to load doxorubicin or irinotecan prior to a TACE procedure [17]). The loading efficacy of irinotecan has already been established [17]. In this study we present in vitro characterisation of DEBTOP, with respect to drug loading capacity, elution characteristics, mechanical properties of the device and some preliminary investigations into efficacy using an in vitro and in vivo pancreatic carcinoma models.

\section{Materials and methods}

\subsection{Materials}

Topotecan hydrochloride powder (Dabur Pharma Ltd., Ghaziabad, India) was dissolved in distilled water $(>17.0$ $\mathrm{M} \Omega$ ) to produce appropriate loading solutions. DC Bead (sulfonate-modified polyvinyl alcohol hydrogel beads from Biocompatibles UK Ltd., Farnham, UK) were used in the size range of 300-500 $\mu \mathrm{m}$ to assess drug loading, elution and cell viability, due to ease of handling compared to the smaller size. $100-300 \mu \mathrm{m}$ beads were used for animal studies as the tumours themselves were only $0.2 \mathrm{~cm}^{3}$ and the smallest available size product was most appropriate for small gauge needle delivery. In order to ensure the beads were retained at the site of injection and not extravasated, they were suspended in a $0.6 \mathrm{wt} / \mathrm{vol}$ alginate solution (ultra-pure Phycomer E01, CellMed AG, Alzenau, 
Germany) which is mannuronic acid rich and has a molecular weight of approximately 800,000 and hence helps to increase viscosity of the suspension. For compressibility studies, DC Bead in the size range of 900-1200 $\mu \mathrm{m}$ were used as these produce the most consistent mechanical data. Solutions used in loading and elution analysis included a 50:50 mix (v/v) of ethanol (Romil Ltd., UK): saturated potassium chloride solution (Fisher Scientific UK Ltd., UK) and phosphate buffered saline solution (PBS) (Inverclyde Biologicals, UK) solutions respectively.

\subsection{Preparation of DEBTOP and determination of drug loading level}

DEBTOP was prepared by removing the packing solution from $1 \mathrm{ml}$ of $300-500 \mu \mathrm{m}$ DC Bead and then adding the drug solution to the vial containing the bead slurry. In order to assess the maximum loading capacity, DC Bead was exposed to topotecan solutions containing between 2 and $100 \mathrm{mg}$ for $3 \mathrm{~h}$. The vials were agitated on a roller mixer. To determine total dose loaded, the loaded beads were removed from their solution, transferred to $500 \mathrm{ml}$ of 50:50 mix of ethanol: saturated $\mathrm{KCl}$ solution and stirred at $700 \mathrm{rpm}$ for $7 \mathrm{~h}$. Use of this rapid-elution medium ensured that all drug was extracted from the beads [12]. This was confirmed by use of HPLC analysis of the extraction solution and mass balance comparison with any residual drug remaining in the loading solution. The HPLC setup comprised of a $\mathrm{C} 18$ reverse phased column and an 85:15 water:acetonitrile with $0.1 \%$ trifluoroacetic acid mobile phase pumped at $1.2 \mathrm{ml} \mathrm{min}^{-1}$ (isocratic). The mobile phase was $<\mathrm{pH} 3$ to ensure that topotecan present was converted to its lactone form to aid analysis. The injection volume was $20 \mu \mathrm{l}$ and the sample was detected using UV absorption at $228 \mathrm{~nm}$.

To assess the effect of agitated loading, beads exposed to $6 \mathrm{mg}$ topotecan $\left(6 \mathrm{ml}, 1 \mathrm{mg} \mathrm{ml}^{-1}\right.$ solution) were either roller-mixed during loading or left static for three hours. A $100 \mu \mathrm{l}$ aliquot of the loading solution was removed from the sample at set time points over three hours and diluted with $900 \mu \mathrm{l}$ of water prior to HPLC analysis as described above.

\subsection{Bead sizing and appearance}

The size and appearance of individual DC Beads during topotecan loading and elution were examined by use of an Olympus BX50F4 microscope with a Colorview III Camera (Olympus, Japan), under an aqueous solution. Sizing data were analysed using AnalySIS (Soft Imaging System $\mathrm{GmbH}$ ) software.

\subsection{Compressibility of DEBTOP}

Analysis of DC Bead compressibility was conducted with 900-1200 $\mu \mathrm{m}$ beads as the technique was less sensitive for smaller beads. DC Bead with and without drug loading under an aqueous solution was assessed using an Instron 4411 with Series IX software (Instron, Canton, MA, USA) as previously described [17]. The general procedure involved distributing the beads onto a specially-fabricated sample holder on the Instron to form a monolayer of about $7-10 \mathrm{~mm}$ in width. The residual water around the beads was carefully removed by wicking using a filter paper. The diameter of the probe used was $5.0 \mathrm{~mm}$ with a crosshead speed of $5.0 \mathrm{~mm} \mathrm{~min}^{-1}$. The load cell used was $50 \mathrm{~N}$ and the specimen gauge length was set as $1.0 \mathrm{~mm}$. All the experiments were carried out at room temperature. The load and compression modulus was calculated from the slope of the curve versus percentage strain at $8-16 \%$ compression strain. Five measurements were performed per sample at room temperature.

\subsection{In vitro release of topotecan into various media}

The elution profiles of DEBTOP were evaluated in PBS at room temperature. Two loading doses were assessed; $6 \mathrm{mg}$ (low) and $30 \mathrm{mg}$ (high) topotecan $\mathrm{ml}^{-1}$ hydrated DC Bead. The loaded beads were then extracted into $500 \mathrm{ml}$ of PBS at room temperature by stirring with a magnetic stirrer at a speed of $700 \mathrm{rpm}$. A $1.5 \mathrm{ml}$ aliquot of the elution solution was removed from the sample at set times over $7 \mathrm{~h}$ and analysed by HPLC. The percentage of topotecan eluted from the beads was then calculated as a function of time.

\subsection{In vitro PSN-1 cytotoxicity}

Human pancreatic adenocarcinoma (PSN-1) cells (purchased frozen from ECACC UK, 94060601) were seeded at a density of 20,000 cells per well (96 well plate) in RPMI (PAA, UK) media containing 10\% Fetal Bovine Serum, (PAA, UK). The cells were incubated under standard conditions $\left(5 \% \mathrm{CO}_{2}, 37^{\circ} \mathrm{C}\right)$ overnight. The media was then removed and replaced with either free topotecan or irinotecan concentrations (0.00001-1000 $\mathrm{g} \mathrm{ml}^{-1}$ media) or a single topotecan or irinotecan loaded DC Bead (6 and $30 \mathrm{mg}$ drug $\mathrm{ml}^{-1} \mathrm{DC}$ Bead) in $200 \mu \mathrm{l}$ of media. An MTS assay (Promega, UK) was used to determine to number of viable cells at 24, 48 and 72 h. Cell viability was expressed as a percentage of control (untreated) cells. Differences in cell viabilities were analysed using a Bonferroni Student's $t$-test with a $P$ value of $<0.05$ being defined as statistically significant. 
2.7 In vivo nude mouse xenograft

Animal studies were performed by Exp. Pharmacol. \& Oncol. GmbH, (Berlin-Buch, Germany) in accordance with local regulations (granted by the LAGeSo, State Office of Health and Social Affairs, Berlin). PSN-1 tumour pieces (human pancreatic carcinoma) were prepared from an in vitro passage for subcutaneous transplantation in female nude mice. Mice were randomised to the scheduled treatment groups ( $n=3$ per group). When the tumours became palpable $\left(0.2 \mathrm{~cm}^{3}\right)$, the DC Bead/alginate mixture was injected close to the subcutaneous growing tumour, using a $1 \mathrm{ml}$ syringe (B.Braun, Germany) fitted with a G16 needle $(0.6 \times 25 \mathrm{~mm}$, Terumo Europe, Belgium $)$. Prior to injection all the lyophilised gamma-sterilised DC Bead (1 ml pre dehydration) were hydrated on the day of administration by the addition of $3 \mathrm{ml}$ alginate solution. The delivery volume was $100 \mu \mathrm{l}$ or $200 \mu \mathrm{l}$ per mouse. In some cases, when the mean tumour size was $\geq 20 \%$ larger than the previous time point a repeat injection was performed at a different site next to the tumour compared to the previous injection. The doses and volumes injected are shown in Table 3. Tumour volumes and body weights were recorded twice weekly. Mice were sacrificed when the tumours reached an average size of $>1 \mathrm{~cm}^{3}$.

\section{Results and discussion}

\subsection{Topotecan loading profiles}

The available topotecan is adsorbed in a linear fashion $\left(R^{2}=0.999\right)$ relative to the loading solution concentration into DC Bead at a dose matching $\approx 88 \%$ of the available drug, up to $35 \mathrm{mg}$ (Fig. 2). The residual salt within the

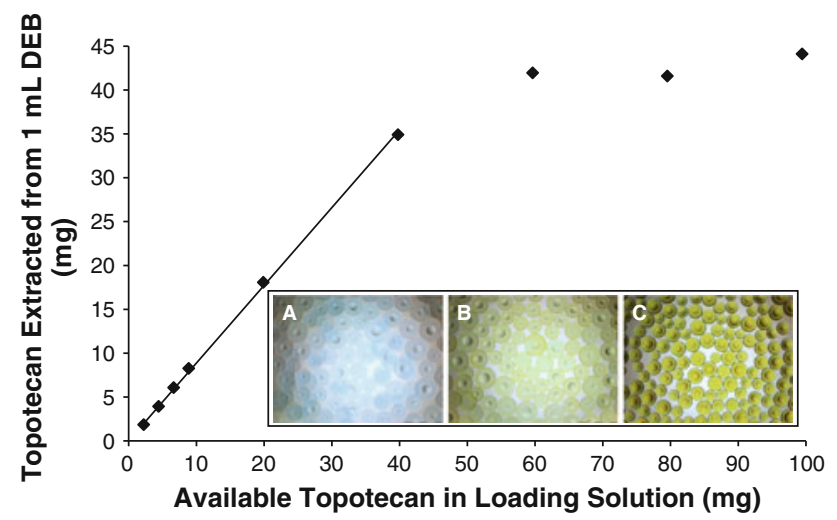

Fig. 2 Topotecan bound to $1 \mathrm{ml}$ of DC Bead compared to the available topotecan. Inset: Light microscopy images $(\times 4$ magnification) of 300-500 $\mu \mathrm{m}$ beads, $(A)$ unloaded in water $(B)$ loaded with $6 \mathrm{mg}$ topotecan and $(C)$ loaded with $30 \mathrm{mg}$ of topotecan packing solution exchanging with ionically bound topotecan was thought to be the reason that all of the available topotecan only loaded to $\approx 88 \%$. The loading appears to plateau at a maximum around $40-45 \mathrm{mg}$ topotecan $\mathrm{ml}^{-1}$ of unloaded DC Bead. These data suggest that all the binding sites are saturated, so even if more topotecan is available it cannot bind to the bead, merely partition into its water fraction. The binding interaction between the drug and bead is attributed to the negatively charged sulfonate group within the bead and the positively charged protonated amine group on the topotecan molecule (Fig. 1). The calculated theoretical number of available binding sites (sulfonate groups) in $1 \mathrm{ml}$ beads is $7.7 \times 10^{-5} \mathrm{~mol}$ [17]. This would equate to a loading of $35 \mathrm{mg}$, which is less than the observed maximum loading. This indicates that more than one topotecan molecule is binding per sulfonate group. This could be achieved by the drug interacting with itself as it becomes concentrated in the bead matrix. It is known in the literature that topotecan does form dimers at high concentrations [18].

It was observed that topotecan partitioned into DC Bead from the $6 \mathrm{mg}$ loading solution faster when the system was agitated compared to stationary loading (Fig. 3). The larger error associated with static loading was attributed to unintentional solution movement when sample was removed for analysis. Agitation helps to disturb the electrical double-layer build up of ions at the beads surface which otherwise impedes the loading process in the static situation. Loading was achieved in $<20 \mathrm{~min}$ when DC Bead was agitated; it is considered that this would be an acceptable time for DC Bead preparation in the pharmacy prior to use.

\subsection{Bead sizing and appearance}

Post topotecan loading, DC Bead exhibited a yellow/green hue, which was more intense upon higher drug loading. This colour was due to a combination of the blue tint of the microsphere and the inherent yellow/green colour of the

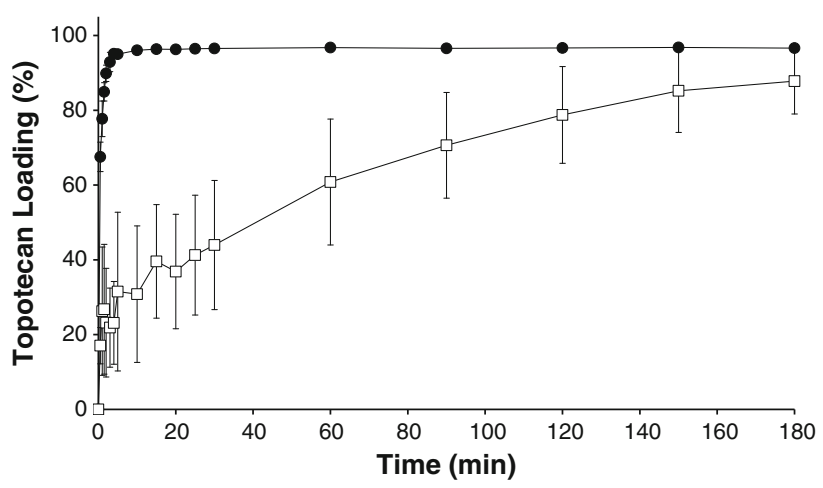

Fig. 3 Loading profile of $6 \mathrm{mg}$ topotecan into DC Bead when agitated or $(\square)$ static $(n=6$, mean $\pm \mathrm{SD})$ 
Table 1 Topotecan loaded $\mathrm{ml}^{-1}$ DC Bead and corresponding diameter $(n=200)$

\begin{tabular}{lll}
\hline Topotecan loaded $(\mathrm{mg})$ & \multicolumn{2}{l}{ Size $(\mu \mathrm{m})$} \\
\cline { 2 - 3 } & Mean & Std. Dev. \\
\hline 0 & 445 & 63 \\
2 & 457 & 73 \\
4 & 427 & 61 \\
6 & 435 & 69 \\
10 & 431 & 57 \\
30 & 340 & 47 \\
\hline
\end{tabular}

drug (Fig. 2, inset). The bead size was shown to decrease with higher topotecan loading (Table 1).

\subsection{Compressibility}

The compression properties of the beads provide an indication of how they will deform when passing through a microcatheter, needle or blood vessel. Compared to the unloaded beads, DC Bead, topotecan loaded exhibited significantly higher compression moduli. Resistance to compression was associated with increased drug loading (Table 2). The increase in compression and decrease in bead size with drug loading were attributed to the hydrophobic nature of the topotecan molecules displacing the water molecules from the hydrogel. Similar phenomena were observed with doxorubicin and irinotecan loaded DC Bead [17]. In addition, the change in compression and bead size may be exaggerated due to the association capabilities of topotecan, which is able to self-aggregate at higher concentrations to form dimers [18]. This may act as extra crosslinking junctions, consequently decreasing the distance between polymer chains (reducing bead size), expelling water and increasing resistance to compression.

\subsection{In vitro drug release}

Topotecan elution is retarded by its interaction with the DC Bead. Figure 4 shows that the elution is dependent on the amount of topotecan loaded. At lower loading $(6 \mathrm{mg})$ topotecan has fully eluted from the DC Bead after $30 \mathrm{~min}$,

Table 2 Topotecan loaded $\mathrm{ml}^{-1}$ DC Bead and corresponding compression modulus $(n=200)$

\begin{tabular}{llc}
\hline Topotecan loaded $(\mathrm{mg})$ & \multicolumn{2}{l}{ Modulus $(\mathrm{kPa})$} \\
\cline { 2 - 3 } & Average & Std. Dev. \\
\hline 0 & 36 & 2 \\
6 & 40 & 2 \\
30 & 95 & 10 \\
\hline
\end{tabular}

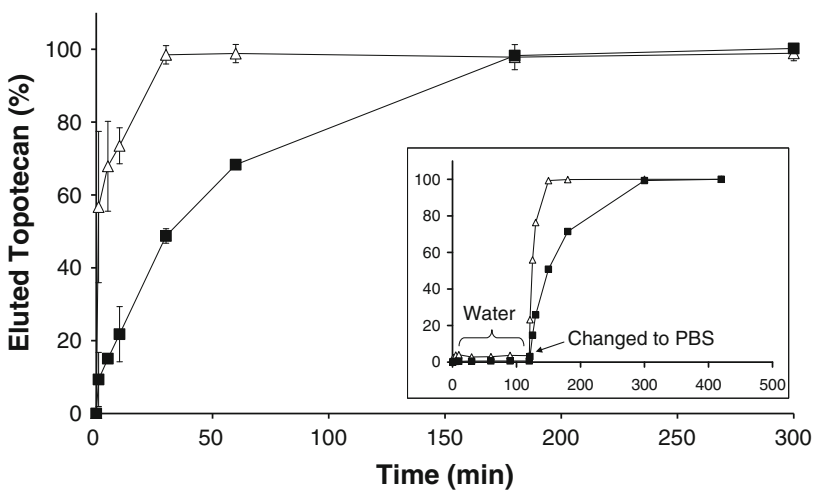

Fig. 4 Elution profiles of $(\Delta) 6 \mathrm{mg}$ and (ם) $30 \mathrm{mg}$ topotecan extracted from 300 to $500 \mu \mathrm{m}$ beads into PBS (\% elution) at room temperature ( $n=6$, mean $\pm \mathrm{SD})$. Inset: ("Elution" of topotecan into water then PBS $(\Delta) 6 \mathrm{mg} \mathrm{ml}^{-1}$ DEBs $\left.(\square) 30 \mathrm{mg} \mathrm{ml}^{-1}(n=1)\right)$

whilst at higher loading $(30 \mathrm{mg})$ the drug takes around $180 \mathrm{~min}$ to fully elute. Topotecan did not elute from DC Bead when the elution medium was water, but was liberated from the beads when the medium was changed to phosphate buffered saline (PBS) (Fig. 4, inset). PBS, a medium more representative of biological fluids, facilitates the release of topotecan through ion exchange; a process not available in distilled water.

The diffusion coefficient, D, for this system was calculated using the method described by Taylor [17]. The D values when $<85 \%$ of the loaded drug was eluted were $2.6 \times 10^{-7}$ and $5.9 \times 10^{-8} \mathrm{~cm}^{2} \mathrm{~s}^{-1}$ for the 6 and $30 \mathrm{mg} \mathrm{ml}^{-1}$ loadings respectively. The diffusion coefficient decreases with increasing drug loading and the difference in topotecan elution can be attributed to the higher hydrophobicity of topotecan or potential dimerisation of topotecan, both resulting in drug-drug interaction and possessing a tighter network through which the drug must permeate.

\subsection{In vitro PSN-1 cytotoxicity}

Pancreatic adenocarcinoma cells were used as a model to test the efficacy of topotecan and irinotecan delivery from DC Bead. Topotecan has been prescribed in patients with advanced pancreatic cancer in clinical models but was not very successful in the dosing regimes attempted. A dose administered as a $30 \mathrm{~min}$ infusion at a dose of $1.5 \mathrm{mg} \mathrm{m}^{-2}$ day $^{-1}$ for five consecutive days every 3 weeks has been shown to exhibit toxicity (neutropenia) and no significant antitumour responses [19]. Alternative dosing regimes at $0.5 \mathrm{mg} \mathrm{m}^{-2}$ day $^{-1}$ over 21 days exhibited $8 \%$ partial response, with reversible and non-cumulative toxicity (myelosuppression) [20]. Localised therapies may offer a method for enhancing efficacy and reducing systemic side effects in vivo and we have shown in the previous sections 
Table 3 Irinotecan hydrochloride and topotecan hydrochloride $\mathrm{IC}_{50}$ for PSN-1 cells

\begin{tabular}{llllll}
\hline Drug & $\mathrm{IC}_{50} \mathrm{mg} \mathrm{ml}^{-1}$ & & & $\mathrm{IC}_{50} \mu \mathrm{M}$ & \\
\cline { 2 - 3 } \cline { 6 - 6 } & $48 \mathrm{~h}$ & $72 \mathrm{~h}$ & & $48 \mathrm{~h}$ & $72 \mathrm{~h}$ \\
\hline Irinotecan & 13 & 19 & & 28.1 & 19.2 \\
Topotecan & 0.101 & 0.123 & & 0.27 & 0.22 \\
\hline
\end{tabular}

that DEBTOP can be easily loaded and the drug eluted in a controlled manner.

Using the MTS assay, both topotecan and irinotecan in their free drug form showed cytotoxicity towards pancreatic adenocarcinoma (PSN-1) cells. The $\mathrm{IC}_{50}$ for irinotecan at $72 \mathrm{~h}$ was $19.2 \mu \mathrm{M}$, slightly higher than the $1-10 \mu \mathrm{M}$ range reported previously [21]. The cytotoxicity of topotecan was greater than irinotecan at the same concentrations for all time points as demonstrated by an $\mathrm{IC}_{50}$ some two orders of magnitude less (Table 3, Fig. 5). The $\mathrm{IC}_{50}$ values obtained for topotecan were not dissimilar to those obtained by other workers using MCF-7 human breast cancer [22] or U87 human glioma cell lines [23], which for comparison were in the range $0.037-0.280 \mu \mathrm{M}$. At earlier time points and lower concentrations both drugs elicited a small increase in cell number, which is thought to be a cellular response to stress. At higher concentrations and later time points, cellular death was observed. Interestingly, at the highest concentration for both drugs at $72 \mathrm{~h}$, the cytotoxicity was slightly less than the corresponding $48 \mathrm{~h}$ data, indicating possible drug-resistance mechanisms may be initiated. When exposed to a single topotecan loaded DC Bead, reduction in PSN-1 cell viability was observed for both 6 and $30 \mathrm{mg} \mathrm{ml}^{-1}$ loadings at statistically greater levels than for the corresponding DEBIRI (Fig. 6). For comparison, DEBIRI exhibited signs of cytotoxicity after 48 and $72 \mathrm{~h}$, but not at $24 \mathrm{~h}$. Topotecan is cytotoxic when

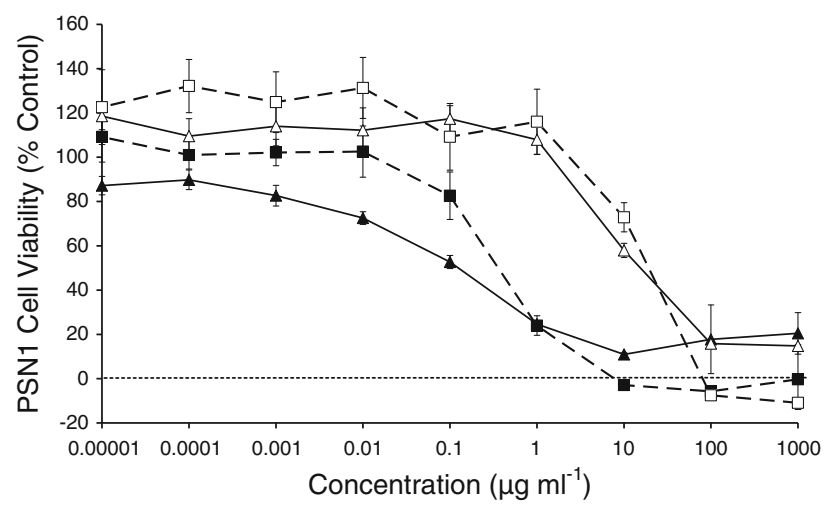

Fig. 5 PSN-1 kill curve for increasing concentrations of free topotecan $(\boldsymbol{\square}) 48 \mathrm{~h}$ and $(\boldsymbol{\Delta}) 72 \mathrm{~h}$ exposure) and free irinotecan $(\square)$ $48 \mathrm{~h}$ and $(\Delta) 72 \mathrm{~h}$ exposure $(n=7$, mean $\pm \mathrm{SD})$

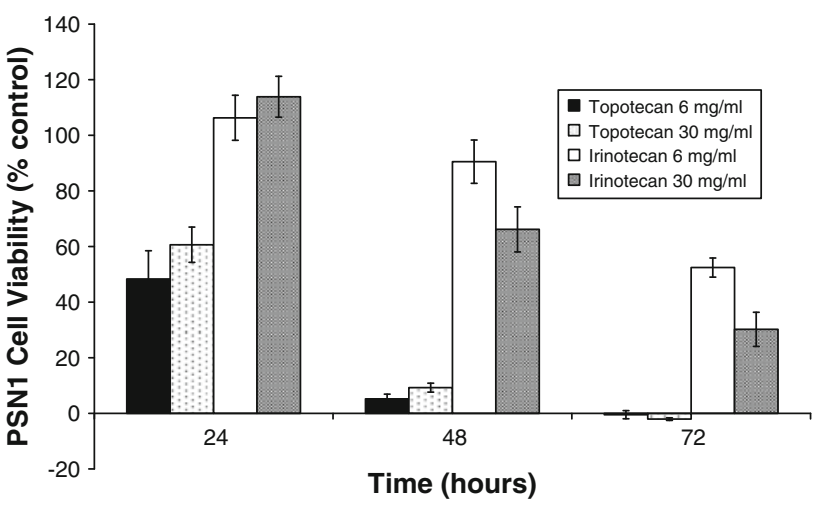

Fig. 6 Cell viability after exposure to a single DEBTOP or DEBIRI for 24,48 and $72 \mathrm{~h}(n=7$, mean $\pm \mathrm{SD})$

Table 4 Group information for mouse xenograft study

\begin{tabular}{lllll}
\hline $\begin{array}{l}\text { Group } \\
\text { type }\end{array}$ & $\begin{array}{l}\text { Bead } \\
\text { loaded per ml of } \\
\text { beads }\end{array}$ & $\begin{array}{l}\text { Total volume } \\
\text { (alginate and DEB) } \\
\text { injected }(\mu \mathrm{l})\end{array}$ & $\begin{array}{l}\text { Dose } \\
(\mathrm{mg})\end{array}$ \\
\hline A & Unloaded & 0 & 100 & 0 \\
B & DEBIRI & 100 & 100 & 3.3 \\
C & & 100 & 200 & 6.6 \\
D & DEBTOP & 37 & 100 & 1.2 \\
E & 25 & 100 & 0.83 \\
F & 12 & 100 & 0.4 \\
G & 6 & 100 & 0.2 \\
\hline
\end{tabular}

eluted from the DC Bead, indicating that topotecan maintains its activity upon elution. The comparison with DEBIRI highlights the greater potency of topotecan despite their matching concentrations.

\subsection{In vivo mouse PSN-1 xenograft response}

The growth of PSN-1 tumours was inhibited with DEBTOP or DEBIRI administered subcutaneously in nude mice (Table 4, Fig. 7). The higher doses of topotecan (1.2 and $0.83 \mathrm{mg}$ ) were lethal to all the mice after six days, whilst the lower concentrations ( 0.4 and $0.2 \mathrm{mg}$ ) showed some efficacy in reducing the tumour volume. The control mice showed severe body weight loss when the tumours reached a larger tumour volume, which was attributed to tumour induced cachexia. Based on this measure of toxicity, all groups (except the toxic higher topotecan doses) showed no significant weight loss, indicating that the treatments were well tolerated.

In agreement with the in vitro results, a much lower dose of topotecan was required to elicit a cytotoxic effect compared to irinotecan. From all of the samples tested here, the higher irinotecan dose showed the greatest potential for survival for 57 days post the tumour implant. 

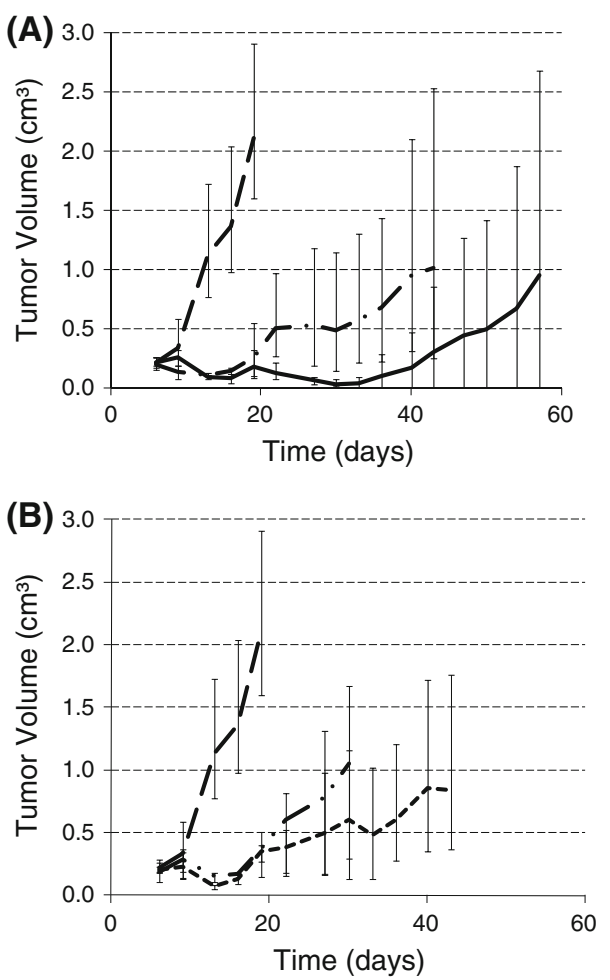

Fig. 7 Tumour volume change for PSN-1 subcutaneous transplantation in female nude mice when treated with a DEBIRI $(-.3 .3 \mathrm{mg}$; $-6.6 \mathrm{mg}$ ) B) DEBTOP ( - $0.2 \mathrm{mg} ; \cdots 0.4 \mathrm{mg})$. The control ( - - ) in both cases were unloaded beads in alginate solution. Repeat injections were made for $3.3 \mathrm{mg}$ DEBIRI (22 and 36 days); $6.6 \mathrm{mg}$ DEBIRI (19 days); $0.4 \mathrm{mg}$ DEBTOP (19 and 27 days) and $0.2 \mathrm{mg}$ DEBTOP (19, 27 and 36 day). ( $n=3$, mean \pm max/min)

Two mice from this group demonstrated significant tumour shrinkage, with one demonstrating $>99 \%$ shrinkage after 47 days and no signs of regrowth after 57 days. One mouse in this group did demonstrate significant tumour growth after 36 days. The variability in the groups may be due to inconsistencies in the exact location of the injection site relative to the tumour; moreover, the tumours grow heterogeneously and sometimes have a capsule, areas with necrotic tissue or larger blood vessels, the variation from which will have been further exacerbated by the low number of animals per group. In the higher irinotecan dose treatment, an increase in tumour size was observed after 19 days. Whilst this could either be due to drug resistance or insufficient drug, the reduction in tumour size after the second injection demonstrates that the initial growth is likely due to the latter effect (Fig. 7).

\section{Conclusions}

In summary, topotecan can be easily loaded into DC Bead within 20 min when the solution and beads are agitated.
The process is slower without agitation as the loading is reliant upon diffusion. As has been seen with doxorubicin and irinotecan, loading with higher concentrations of topotecan changed the colour as well as size and compressibility of the DC Bead. These factors changed within the expected range and were markedly different at higher $\left(30 \mathrm{mg} \mathrm{ml}^{-1}\right.$ ) concentrations. The release from $1 \mathrm{ml} \mathrm{DC}$ Bead took 30 or 180 min to fully elute in a PBS sink environment for 6 and $30 \mathrm{mg}$ topotecan loading respectively. Topotecan efficacy against human pancreatic carcinoma cells was demonstrated in vitro both as a free drug and from DEBTOP, where it was shown to be more potent with an $\mathrm{IC}_{50}$ two orders of magnitude lower than DEBIRI. Both irinotecan and topotecan demonstrate the capacity to be loaded and eluted from DC Bead and show efficacy in a nude mouse human pancreatic adenoma xenograft model. The best treatment (DEBIRI) reduced the tumour by $>99 \%$ in one mouse, extending the average time it took for the tumour to become $1 \mathrm{~cm}^{3}$ in size from 13 to 57 days and demonstrated the feasibility and tolerability of repeat direct injections. Higher doses of topotecan (0.83-1.2 mg) however, were lethal at 6 days post injection, which serves to highlight that dose selection is still critical when considering the balance between efficacy and severe toxicity/ death. Overall, the treatment with either DEBIRI or DEBTOP was well tolerated and could be considered as a method to treat pancreatic cancer either through direct injection or via arterial administration.

Open Access This article is distributed under the terms of the Creative Commons Attribution Noncommercial License which permits any noncommercial use, distribution, and reproduction in any medium, provided the original author(s) and source are credited.

\section{References}

1. Beretta GL, Perego P, Zunino F. Targeting topoisomerase I: molecular mechanisms and cellular determinants of response to topoisomerase I inhibitors. Expert Opin Ther Targets. 2008; 12(10):1243-56.

2. Potmesil M. Camptothecins: from bench research to hospital wards. Cancer Res. 1994;54(6):1431-9.

3. Malagari K. Drug-eluting particles in the treatment of HCC: chemoembolization with doxorubicin-loaded DC Bead. Expert Rev Anticancer Ther. 2008;8(10):1643-50.

4. Kettenbach J, Stadler A, Katzler IV, Schernthaner R, Blum M, Lammer J, Rand T. Drug-loaded microspheres for the treatment of liver cancer: review of current results. Cardiovasc Intervent Radiol. 2008;31(3):468-76.

5. Poon RT, Tso WK, Pang RW, Ng KK, Woo R, Tai KS, Fan ST. A phase I/II trial of chemoembolization for hepatocellular carcinoma using a novel intra-arterial drug-eluting bead. Clin Gastroenterol Hepatol. 2007;5(9):1100-8.

6. de Baere T, Deschamps F, Teriitheau C, Rao P, Conengrapht K, Schlumberger M, Leboulleux S, Baudin E, Hechellhammer L. Transarterial chemoembolization of liver metastases from well differentiated gastroenteropancreatic endocrine tumors with 
doxorubicin-eluting beads preliminary results. J Vasc Interven Radiol. 2008;19(6):855-61.

7. Aliberti C, Tilli M, Benea G, Fiorentini G. Trans-arterial chemoembolization (TACE) of liver metastases from colorectal cancer using irinotecan-eluting beads: preliminary results. Anticancer Res. 2006;26(5):3793-5.

8. Fiorentini G, Aliberti C, Turrisi G, Del Conte A, Rossi S, Benea G, Giovanis P. Intraarterial hepatic chemoembolization of liver metastases from colorectal cancer adopting irinotecan-eluting beads: results of a phase II clinical study. In Vivo. 2007;21(6): 1085-91.

9. Keese M, Gasimova L, Schwenke K, Yagublu V, Shang E, Faissner R, Lewis A, Samel S, Löhr M. Doxorubicin and mitoxantrone drug eluting beads for the treatment of experimental peritoneal carcinomatosis in colorectal cancer. Int $\mathbf{J}$ Cancer. 2009;124(11):2701-8.

10. Baltes S, Freund I, Lewis AL, Nolte I, Brinker T. Doxorubicin and irinotecan drug-eluting beads for treatment of glioma: a pilot study in a rat model. J Mater Sci: Mater Med. 2010;21(4): 1393-402.

11. Lewis AL. DC Bead: a major development in the toolbox for the interventional oncologist. Expert Rev Med Devices. 2009;6(4): 389-400.

12. Gonzalez MV, Tang Y, Phillips GJ, Lloyd AW, Hall B, Stratford PW, Lewis AL. Doxorubicin eluting beads-2: methods for evaluating drug elution and in vitro: in vivo correlation. J Mater Sci: Mater Med. 2008;19(2):767-75.

13. Lewis AL, Gonzalez MV, Leppard SW, Brown JE, Stratford PW, Phillips GJ, Lloyd AW. Doxorubicin eluting beads-1: effects of drug loading on bead characteristics and drug distribution. J Mater Sci: Mater Med. 2007;18(9):1691-9.

14. Tang Y, Czuczman PR, Chung ST, Lewis AL. Preservation of the active lactone form of irinotecan using drug eluting beads for the treatment of colorectal cancer metastases. J Control Release. 2008;127(1):70-8.
15. Chu E, Sartorelli AC. Cancer chemotherapy. In: Katzung BG, editor. Basic \& clinical pharmacology. McGraw-Hill Professional, 2007; 2007.

16. Lesimple T, Hassel MB, Gedouin D, Seigneuret E, Carsin B, Hamlat A, Riffaud L, Simon H, Malhaire JP, Guegan Y. Phase I study of topotecan in combination with concurrent radiotherapy in adults with glioblastoma. J Neurooncol. 2003;65(2):141-8.

17. Taylor RR, Tang Y, Gonzalez MV, Stratford PW, Lewis AL. Irinotecan drug eluting beads for use in chemoembolization: in vitro and in vivo evaluation of drug release properties. Eur $\mathbf{J}$ Pharm Sci. 2007;30(1):7-14.

18. Strel'tsov SA, Grokhovskii SL, Kudelina IA, Oleinikov VA, Zhuze AL. [Interaction of topotecan - a DNA topoisomerase inhibitorwith dual-stranded polydeoxyribonucleotides. I. Dimerization of topotecan in solution]. Mol Biol (Mosk). 2001;35(3):432-41.

19. O'Reilly S, Donehower RC, Rowinsky EK, Ord S, Grochow LB. A phase II trial of topotecan in patients with previously untreated pancreatic cancer. Anticancer Drugs. 1996;7(4):410-4.

20. Stevenson JP, Scher RM, Kosierowski R, Fox SC, Simmonds M, Yao KS, Green F, Broom C, Fields SZ, Krebs JB, et al. Phase II trial of topotecan as a 21-day continuous infusion in patients with advanced or metastatic adenocarcinoma of the pancreas. Eur $\mathbf{J}$ Cancer. 1998;34(9):1358-62.

21. Saito Y, Yasunaga M, Kuroda J, Koga Y, Matsumura Y. Enhanced distribution of NK012, a polymeric micelle-encapsulated SN-38, and sustained release of SN-38 within tumors can beat a hypovascular tumor. Cancer Sci. 2008;99(6):1258-64.

22. Akbas SH, Timur M, Ozben T. The effect of quercetin on topotecan cytotoxicity in MCF-7 and MDA-MB 231 human breast cancer cells. J Surg Res. 2005;125(1):49-55.

23. Pollina J, Plunkett RJ, Ciesielski MJ, Lis A, Barone TA, Greenberg SJ, Fenstermaker RA. Intratumoral infusion of topotecan prolongs survival in the nude rat intracranial U87 human glioma model. J Neurooncol. 1998;39(3):217-25. 